\title{
The risk of early complications in patients with hand infections
}

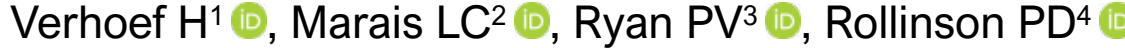

$1 \mathrm{MBChB}(U P)$; Orthopaedic Registrar, Department of Orthopaedic Surgery, Nelson R Mandela School of Clinical Medicine, University of KwaZulu-Natal, Durban, South Africa

2 MBChB, FC Orth SA, MMed(Orth), PhD; Head of Department, Department of Orthopaedic Surgery, School of Clinical Medicine, University of KwaZulu-Natal, Durban, South Africa

3 MBChB(UCT), H Dip Orth(SA); FCS Orth(SA); MMed(SA); HOD Orthopaedic Surgery, Inkosi Albert Luthuli Central Hospital; University of KwaZulu-Natal, Durban, South Africa

4 MBChB(Sheff), FRCS (Ed \& Eng); Chief Orthopaedic Specialist, Ngwelezana Hospital

Corresponding author: Dr Hein Verhoef, Unit 19 El Torero, 86 Lewis Drive, Amanzimtoti, Durban, 4126; tel: 082 929 544; email: heinkvkp@hotmail.com

\section{Abstract}

Background: The aim of this study was to identify the risk factors associated with the development of early complications in patients with hand infections. A secondary objective was to describe the bacteriology and resistance profile in our study population.

Methods: This retrospective observational descriptive study was performed at a regional referral centre in South Africa. All primary hand infection cases treated over a period of one year were reviewed. Children under 18 years, cases with incomplete primary outcome data and post-operative infections were excluded. Clinical and demographic data was extracted from clinical records. Amputation, re-debridement and tissue loss requiring skin grafting were regarded as early complications. Bacteriological analysis comprised identification of causative organisms as well as evaluation of their resistance profiles. Risk factors that were found to be significant for development of early complications were entered into a multivariate regression analysis.

Results: After inclusion and exclusion criteria were applied, 78 patients were deemed eligible for inclusion to the study. The patientassociated risk factors that were found to be associated with the development of early complications after univariate analysis were increasing age and poorly controlled diabetes mellitus. Human bites and polymicrobial infections were the only aetiological factors that were identified to be significant on a univariate level. Initial presentation to a private sector general practitioner (GP) was the only management factor to reach significance on univariate analysis. Human immunodeficiency virus (HIV) infection, CD4 count, viral load and duration of ARV treatment were not found to be significantly associated with the development of early infections. On multivariate logistic regression analysis, poorly controlled diabetes mellitus, human bites and first presentation to a private GP were the only risk factors that remained significant for the development of early complications. The culture yield was $68 \%$. Staphylococcus aureus (S. aureus) was the most frequently isolated organism (37\%), followed by polymicrobial infections (10\%). S. aureus encountered in our study population remained mostly sensitive to cloxacillin; however, high levels of resistance (50\%) to ampicillin were observed. Klebsiella sp. and Acinetobacter sp. were the most frequently observed Gram-negative organisms.

Conclusion: After multivariate regression analysis, hand infections in poorly controlled diabetic patients, infections occurring after human bites as well as those affected by polymicrobial infections were identified as independent risk factors for development of early complications in patients with hand infections. HIV infection was not found to be a significant risk factor. Our bacteriological profile is in keeping with trends demonstrated in the literature where $S$. aureus infections seem to be declining in frequency while polymicrobial infections seem to be encountered more frequently.

Level of evidence: Level 4

Keywords: HIV, diabetes mellitus, hand infection, complication, risk factor

Citation: Verhoef H, Marais LC, Rollinson PD, Ryan PV. The risk of early complications in patients with hand infections. SA Orthop J 2020;19(2):97102. http://dx.doi.org/10.17159/2309-8309/2020/v19n2a7

Editor: Prof. Theo le Roux, University of Pretoria, South Africa

Received: July $2019 \quad$ Accepted: January $2019 \quad$ Published: May 2020

Copyright: $\odot 2020$ Verhoef $\mathrm{H}$. This is an open-access article distributed under the terms of the Creative Commons Attribution Licence, which permits unrestricted use, distribution and reproduction in any medium, provided the original author and source are credited.

Funding: No funding was received for this study.

Conflict of interest: The authors declare that they have no conflicts of interest that are directly or indirectly related to the research. 


\section{Introduction}

Acute hand infections are common and despite the advances in antibiotic therapy and surgical interventions, remain a challenging clinical entity to treat. ${ }^{1}$ Hand infections can be associated with significant morbidity and may result in marked permanent physical impairment, especially if appropriate management is not initiated timeously. ${ }^{1-3} \mathrm{~A}$ multitude of factors have been suggested to influence the development, bacteriology and outcomes of hand infections.

Predisposing factors for the development of hand infections that have been identified include intravenous drug use, diabetes mellitus, steroid use and other immune-compromising conditions. ${ }^{4}$ Attempts have been made to study the association between human immunodeficiency virus (HIV) and hand infections, but these studies either had small numbers of HIV-positive patients or CD4 counts were not routinely performed or available.,.$^{2,5}$ Diabetes mellitus has been shown to predispose to the development of infectious conditions and has also been associated with poor outcomes in infections of the hand. ${ }^{8}$ Other factors that have been suggested to alter the course and outcome of hand infections include the employment status; nature of occupation; the aetiology, nature and site of infection; metabolic and nutritional status; as well as the age and sex of patients. ${ }^{2,9}$ The variability of reported factors suggests that multiple factors may be interacting in individual patients; however, multivariate analyses seem to indicate shortcomings in many of the reported studies including the most frequently cited publications. $^{10}$

Intravenous antibiotic therapy is an important component of early management and initially this takes the form of generic treatment based on the most likely causative organisms. Historically Staphylococcus aureus (S. aureus) was the most commonly isolated organism, but more recent literature suggests that this trend may be changing. ${ }^{11}$ Furthermore, the bacteriology may also vary in different patients with different risk factors. Therefore, knowledge of local microorganism and antibiotic resistance profiles may be useful in determining an optimal initial antibiotic strategy.

The aim of this study was to identify the risk factors associated with the development of early complications in patients with hand infections. A secondary objective was to describe the bacteriology and resistance profile in our study population.

\section{Methods}

This retrospective observational study was performed in a regional hospital which is a referral centre for 15 district level hospitals in a predominantly rural and semi-rural area of KwaZulu-Natal. All patients treated for primary hand infections between July 2016 and July 2017 were deemed eligible for inclusion in the study. Patients younger than 18 years, cases with incomplete primary outcome data and cases involving post-operative infections were excluded. Patients who presented initially with clean traumatic hand wounds or fractures requiring open reduction and internal fixation, tendon repair, nerve repair, external splinting in the form of plaster cast or external fixator, who subsequently developed hand infections were also excluded.

Data relating to the following demographic and clinical variables were extracted from clinical records: age, sex, hand dominance, diabetic and HIV status, HbA1c levels in diabetic patients, CD4 counts and viral load in HIV-positive patients, source of infection, onset and presentation, occupation, surgical procedures performed and duration of hospital stay. Pus swabs were analysed to identify the causative organism and to determine patterns of sensitivity to antibiotics. The need for re-operation, amputation and need for skin grafting due to tissue loss were regarded as complications.
The standard management protocol included facilitation of prompt transfer for patients with suspected hand infections from referring hospitals. HIV ELISA testing was performed on all patients unless the patient was known to be HIV-positive and already on anti-retroviral (ARV) treatment. CD4 count and HIV viral load was performed on all HIV-positive patients and $\mathrm{HbA1C}$ level on all diabetic patients. Pus swabs were taken in theatre for microscopy, culture and sensitivity (MCS) testing. Empiric antibiotic administration included intravenous (IV) cloxacillin $1 \mathrm{~g}$ 8 hourly (or second-generation cephalosporin if cloxacillin was not available) for all patients. Gentamycin $240 \mathrm{mg}$ IV daily and metronidazole $500 \mathrm{mg}$ IV 8 hourly were added for HIV-positive and diabetic patients. Empiric intravenous antibiotics were commenced on admission and continued until the culture and sensitivity results became available, and then culture-directed antibiotic therapy was instituted. Daily paraffin gauze or absorbent dressings were done throughout admission. Patients were discharged on oral antibiotics once the drainage had sufficiently decreased and further surgical intervention in the form of re-debridement, amputation or skin grafting was deemed unlikely. Patients were referred to occupational therapists for rehabilitation once pain and wound drainage allowed. Daily dressings and rehabilitation continued on an outpatient basis at our institution, or at the referral hospital.

Statistical analysis was performed using Stata 15.0 (StataCorp. College Station, Texas). Continuous variables were reported as mean ( \pm standard deviation [SD]) or median (with interquartile range $[\mathrm{IQR}]$ ) and categorical variables as numbers and percentages, unless otherwise stated. Differences in continuous variables were compared with the use of the Mann-Whitney test. Categorical data was compared using the Fisher's exact test (if any cell count was below 5) or the chi-squared test (if no cell count below 5). All tests were two-sided and the level of significance was set at $p<0.05$. In addition, reverse stepwise logistic regression analysis was performed and factors that were significantly associated with early complications were then used in the multivariate regression model.

\section{Results}

A total of 105 cases of primary hand infection cases were identified over the study period. Two cases with incomplete primary outcome data and 25 cases involving patients under the age of 18 years were excluded from the study. Therefore, 78 cases were included in the analysis. The demographic information of the study population is summarised in Table I. The median age of patients was $38.8 \pm 15.6$ years (range 18-90 years) of which $44(56 \%)$ were males. The majority of patients $(60 \%)$ were unemployed and $22 \%$ were manual labourers. Sixty-eight patients $(91 \%)$ were right-handed and the dominant hand was affected in $54 \%$ of all patients. In terms of the aetiology, $39(50 \%)$ of hand infections developed spontaneously, $23(30 \%)$ following penetrating injuries and seven (9\%) as the result of human bites.

\section{Risk factors for early complications}

Thirty per cent of patients developed early complications with $14(18 \%)$ cases requiring re-debridement, 12 (16\%) needing amputation and eight (10\%) patients having tissue loss requiring skin graft. Increasing age was associated with a risk for the development of an early complication $(p=0.040)$. The mean time from onset to presentation at the hospital was $6.4 \pm 5.6$ days (range 0-29) and a delay in presentation was not associated with an increased risk for complications $(p=0.810)$. Patients whose first healthcare contact was with a private general practitioner (GP) were, however, at risk for early complications $(p=0.013)$. Only five $(45 \%)$ of these cases were referred for surgical management 
Table I: Description of the study population

\begin{tabular}{|c|c|c|}
\hline & n (\%) & Mean ( \pm SD) \\
\hline Total & 78 (100\%) & \\
\hline Age (years) & & $38.8( \pm 15.6)$ \\
\hline Male sex & $44(56 \%)$ & \\
\hline \multicolumn{3}{|l|}{ Employment status } \\
\hline Unemployed & $47(60 \%)$ & \\
\hline Manual labourer & $17(22 \%)$ & \\
\hline Other & $14(18 \%)$ & \\
\hline Right hand dominant & $68(91 \%)$ & \\
\hline Dominant hand affected & $42(54 \%)$ & \\
\hline HIV positive & $34(43 \%)$ & \\
\hline CD4 count cells $/ \mathrm{mm}^{3}$ & & $400.0( \pm 218.3)$ \\
\hline CD4 count $<350$ cells $/ \mathrm{mm}^{3}$ & $13(38 \%)$ & \\
\hline Viral load undetectable & $9(27 \%)$ & \\
\hline ARV treatment $>3$ months & $14(41 \%)$ & \\
\hline Diabetes mellitus (DM) & 15 (19\%) & \\
\hline $\mathrm{HbA1C}(\%)$ & & $10.69( \pm 4.3)$ \\
\hline Patients on therapy & $12(80 \%)$ & \\
\hline $\mathrm{HbA} 1 \mathrm{C}>6 \%$ & $15(100 \%)$ & \\
\hline HIV and DM & $5(6 \%)$ & \\
\hline Corticosteroid therapy & $0(0 \%)$ & \\
\hline Cigarette smoking & $13(17 \%)$ & \\
\hline Chronic renal failure & $1(1 \%)$ & \\
\hline \multicolumn{3}{|l|}{ Aetiology } \\
\hline Spontaneous onset & $39(50 \%)$ & \\
\hline Penetrating trauma & $23(30 \%)$ & \\
\hline Blunt trauma & $9(11 \%)$ & \\
\hline Human bite & $7(9 \%)$ & \\
\hline \multicolumn{3}{|l|}{ Causative organisms } \\
\hline Staphylococcus aureus & $29(37 \%)$ & \\
\hline Other Gram positives & $4(5 \%)$ & \\
\hline Gram negatives & $12(16 \%)$ & \\
\hline Polymicrobial & $8(10 \%)$ & \\
\hline No growth & $25(31 \%)$ & \\
\hline \multicolumn{3}{|l|}{ Initial healthcare contact } \\
\hline Public clinic & $45(58 \%)$ & \\
\hline Public hospital & $20(26 \%)$ & \\
\hline Private GP & $11(14 \%)$ & \\
\hline Other & $1(1 \%)$ & \\
\hline Onset to presentation (days) & & $6.4( \pm 5.6)$ \\
\hline Length of hospital stay (days) & & $6.9( \pm 6.1)$ \\
\hline Any complication & $23(30 \%)$ & \\
\hline Re-debridement required & $14(18 \%)$ & \\
\hline Amputation & $12(16 \%)$ & \\
\hline Tissue loss requiring skin graft & $8(10 \%)$ & \\
\hline
\end{tabular}

at the time of initial presentation, with the number of healthcare provider contacts prior to formal surgical treatment ranging from one to six. Three patients were given antibiotics and were not referred for surgery, while another three received informal incision and drainage at initial contact. Early complications were seen in four out of the six patients who were not referred at initial contact.
Seven (64\%) complications were seen in patients who were initially treated by a private GP, including five (45\%) amputations, one repeat debridement, and one patient requiring skin grafting due to tissue loss. All three patients who had informal incision and drainage subsequently required an amputation.

Thirty-four (43\%) patients were HIV positive, with a mean CD4 count of $400 \pm 218$ cells $/ \mathrm{mm}^{3}$ and $41 \%$ of cases were on anti-retroviral (ARV) therapy for longer than three months at the time of presentation. The median viral load was 50 copies $/ \mathrm{ml}$ (IQR 0-90 000) and 27\% of cases had an undetectable viral load. HIV-positive status was not associated with an increased risk for early complications $(p=0.565)$. Neither CD4 count, viral load nor ARV therapy were associated with an increased risk of complications (Table II). Eighty per cent of diabetic HIV-positive patients developed early complications $(p=0.025)$. Nineteen per cent of the entire cohort was diabetic with a mean $\mathrm{HbA} 1 \mathrm{c}$ $10.7 \pm 4.3 \%$ (range $6.6-15.6 \%$ ). Forty-three per cent of diabetic patients developed early complications $(p=0.012)$. Infections resulting from a human bite were more likely to develop an early complication than spontaneous cases or cases resulting from penetrating injuries $(p=0.018)$. Polymicrobial infections were also associated with early complications $(p=0.045)$.

Backwards stepwise logistic regression analysis found diabetes mellitus (odds ratio [OR] 4.4, 95\% confidence interval [Cl] 1.12-17.38), human bites (OR 7.6, 95\% Cl 1.15-49.92) and first healthcare contact with a private GP (OR 6.7, 95\% Cl 1.52-29.21) to be associated with an increased risk for early complications (Table III). These factors remained significant on multivariate regression analysis. A post-hoc power analysis revealed that the study had a $95 \%$ power for human bites but was insufficiently powered for diabetes mellitus and first contact with private general practitioner.

\section{Bacteriology and resistance profiles}

Our culture yield was $68 \%(n=53)$. Forty-five swabs $(58 \%)$ cultured a single organism, while no organism was cultured in 25 (32\%) patients. Two organisms were identified in three swabs and one swab cultured three organisms. On four occasions, culture results were reported as mixed growth, and were not further reported. In $29(37 \%)$ cases, S. aureus was identified as a causative pathogen (Table IV). The $S$. aureus organisms cultured showed high levels of resistance to ampicillin (50\%) and only one instance of resistance to cloxacillin was observed. This specific organism also showed intermediate resistance to ciprofloxacin. Another S. aureus organism showed intermediate resistance to vancomycin, but the majority of $S$. aureus cultures remained sensitive to the tested antibiotics. Four infections were caused by other Gram-positive organisms, three being Enterococcus spp. and one Staphylococcus lentus. Nine infections involved Enterobacteriaceae (Klebsiella pneumonia, Klebsiella oxycota, Proteus mirabilis, Enterobacter cloacae and Serratia marcescens). The remaining three Gram-negative infections involved Pseudomonas spp., Acinetobacter spp. and Citrobacter spp. Resistance was most frequently observed among Klebsiella and Acinetobacter spp. The Klebsiella spp. showed the widest range of resistance and were resistant to ampicillin in all instances. Both Acinetobacter spp. and Klebsiella spp., however, remained mostly sensitive to gentamycin and ciprofloxacin in our study population (Table IV).

\section{Discussion}

This study aimed to identify risk factors for the development of early complications after hand infections. The only patient factor that increased the risk for the development of early complications was 
Table II: Risk factors associated with early complications in hand infections

\begin{tabular}{|c|c|c|c|}
\hline Risk factor & No complication n (\%) & Any complication $\mathrm{n}(\%)$ & p-value \\
\hline \multicolumn{4}{|l|}{ Patient factors } \\
\hline Age & $55(70 \%)$ & $23(30 \%)$ & 0.040 \\
\hline Male sex & $34(77 \%)$ & $10(33 \%)$ & 0.136 \\
\hline Unemployed & $30(64 \%)$ & $17(36 \%)$ & 0.111 \\
\hline Dominant hand affected & $28(67 \%)$ & $14(33 \%)$ & 0.391 \\
\hline HIV positive & $22(67 \%)$ & $11(33 \%)$ & 0.565 \\
\hline CD4 count & $23(67 \%)$ & $11(33 \%)$ & 0.755 \\
\hline Viral load & $22(76 \%)$ & $7(24 \%)$ & 0.836 \\
\hline ARV therapy $<3$ months & $12(85 \%)$ & $2(15 \%)$ & 0.390 \\
\hline Diabetes mellitus & $6(43 \%)$ & $9(57 \%)$ & 0.012 \\
\hline HbA1C & $6(43 \%)$ & $8(57 \%)$ & 0.747 \\
\hline HIV positive and diabetes mellitus & $1(20 \%)$ & $4(80 \%)$ & 0.025 \\
\hline Cigarette smoking & 9 (69\%) & $4(31 \%)$ & 1.000 \\
\hline \multicolumn{4}{|l|}{ Aetiological factors } \\
\hline Spontaneous onset & $31(82 \%)$ & $7(18 \%)$ & 0.052 \\
\hline Penetrating trauma & $17(70 \%)$ & $6(30 \%)$ & 0.753 \\
\hline Human bite & $2(29 \%)$ & $5(71 \%)$ & 0.018 \\
\hline Staphylococcus aureus & $25(86 \%)$ & $4(14 \%)$ & 0.078 \\
\hline Other Gram-positive organisms & $2(50 \%)$ & $2(50 \%)$ & 0.577 \\
\hline Gram-negative organisms & $9(75 \%)$ & $3(25 \%)$ & 1.000 \\
\hline Polymicrobial infections & $3(37 \%)$ & $5(63 \%)$ & 0.045 \\
\hline No growth & $17(68 \%)$ & $8(32 \%)$ & 0.738 \\
\hline \multicolumn{4}{|l|}{ Management factors } \\
\hline Initial healthcare contact public clinic & $35(78 \%)$ & $10(22 \%)$ & 0.100 \\
\hline Initial healthcare contact public hospital & $14(70 \%)$ & $6(30 \%)$ & 0.953 \\
\hline Initial healthcare contact private GP & $4(36 \%)$ & $7(64 \%)$ & 0.013 \\
\hline Delay in presentation & $55(71 \%)$ & $23(29 \%)$ & 0.810 \\
\hline
\end{tabular}

Table III: Multivariate regression analysis of risk factors associated with the development of early complications

\begin{tabular}{|l|c|c|c|}
\hline Risk factor & Odds ratio & p-value & $95 \% \mathbf{C l}$ \\
\hline Diabetes mellitus & 4.4 & 0.034 & $1.12-17.38$ \\
\hline Human bite & 7.6 & 0.035 & $1.15-49.92$ \\
\hline First healthcare contact private GP & 6.7 & 0.012 & $1.52-29.21$
\end{tabular}

Table IV: Bacteriology and antibiotic resistance profiles (Percentages indicate the fraction of all organisms found to be either sensitive or resistant to the antibiotics in question; eight infections [10\%] involved multiple organisms; pathogens not listed here)

\begin{tabular}{|c|c|c|c|}
\hline Pathogen & n (\%) & Antibiotic sensitivity (\%) & Antibiotic resistance (\%) \\
\hline Staphylococcus aureus & $29(37 \%)$ & $\begin{array}{l}97 \% \text { - Cloxacillin } \\
100 \% \text { - Clindamycin }\end{array}$ & $50 \%$ - Ampicillin \\
\hline Staphylococcus lentus & $1(<1 \%)$ & $100 \%$ - Cloxacillin, gentamycin, ciprofloxacin & $100 \%$ - Tetracycline \\
\hline Enterococcus sp. & $3(4 \%)$ & 100\% - Ampicillin & $\begin{array}{l}33 \% \text { - Clindamycin } \\
67 \% \text { - Erythromycin, tetracycline }\end{array}$ \\
\hline Klebsiella sp. & $5(6 \%)$ & $80 \%$ - Gentamycin, ciprofloxacin, cefuroxime & $\begin{array}{l}20 \% \text { - Ciprofloxacin, gentamycin, amoxicillin/ } \\
\text { clavulanic acid, all cephalosporins, piperacillin/ } \\
\text { tazobactam } \\
100 \% \text { - Ampicillin }\end{array}$ \\
\hline Proteus sp. & $2(3 \%)$ & $\begin{array}{l}100 \% \text { - Ampicillin, ciprofloxacin, gentamycin, amoxicillin/ } \\
\text { clavulanic acid }\end{array}$ & $100 \%$ - Tigecycline, colistin \\
\hline Serratia sp. & $1(<1 \%)$ & $100 \%$ - Ciprofloxacin, gentamycin & $100 \%$ - Ampicillin, nitrofurantoin, chloramphenicol \\
\hline Enterobacter sp. & $1(<1 \%)$ & $\begin{array}{l}100 \% \text { - Ciprofloxacin, gentamycin, amoxicillin/clavulanic } \\
\text { acid }\end{array}$ & $100 \%$ - Cefuroxime, cefoxitin \\
\hline Pseudomonas sp. & $1(<1 \%)$ & $\begin{array}{l}100 \% \text { - Amoxicillin/clavulanic acid, ciprofloxacin, } \\
\text { gentamycin }\end{array}$ & 100\% - Colistin \\
\hline Acinetobacter sp. & $1(<1 \%)$ & $100 \%$ - Ciprofloxacin, gentamycin & $\begin{array}{l}100 \% \text { - All cephalosporins, piperacillin/ } \\
\text { tazobactam, meropenem }\end{array}$ \\
\hline Citrobacter sp. & $1(<1 \%)$ & $\begin{array}{l}100 \% \text { - Ciprofloxacin, gentamycin, amoxicillin/clavulanic } \\
\text { acid, piperacillin/tazobactam }\end{array}$ & Nil resistance found \\
\hline No growth & $25(32 \%)$ & & \\
\hline Polymicrobial & $8(10 \%)$ & & \\
\hline
\end{tabular}


the presence of diabetes mellitus (OR 4.4). Neither HIV infection nor a low CD4 count was associated with early complications. Patients with infections resulting from human bites had a seven times higher risk for early complications. Patients who were initially treated in the private sector by a GP also had an increased risk for early complications (OR 6.7).

HIV is a retrovirus that causes immune suppression through suppression of CD4 T-lymphocytes. These cells release cytokines that activate macrophages in response to bound antigens and also stimulate $B$ lymphocytes to divide, differentiate and produce antibodies. ${ }^{10}$ The burden of HIV infection in South Africa is among the highest in the world, with $19 \%$ of the adult population being affected,,$^{12}$ and as much as $40 \%$ in certain regions. ${ }^{12,13}$ There are few publications with original data relating to the effect of HIV on hand infections. Ching et al. suggested that HIV-positive patients required higher numbers of surgical procedures and longer admission. ${ }^{10}$ The authors also found that low CD4 counts were associated with more complications. ${ }^{10}$ Both Gonzales et al. and McAulliffe et al., on the other hand, suggested that HIV infection had little bearing on the outcomes of hand infections.5,6 Wynn et al., however, acknowledged that multiple factors could be contributing to the outcomes of hand infections in HIV-positive patients. ${ }^{14}$ Although our study included higher numbers of HIVpositive patients and CD4 counts than previous publications, with the addition of viral load and ARV treatment data, these factors did not seem to have a significant influence on the development of early complications. Taking the small difference in event rates into account, it appears that far greater numbers of HIV-positive patients would be required in order to adequately power a multivariate analysis of all contributing factors.

Chronic poor glycaemic control in diabetic patients leads to impaired cellular immunity, peripheral neuropathy and peripheral microvascular disease. ${ }^{15}$ Elevated serum glucose may impair neutrophil phagocytosis and chemotaxis. Loss of protective sensory perception to heat, pain and pressure means that diabetic patients may have an altered perception of the severity of trauma or may not notice the effects of repetitive microtrauma such as repetitive manual labour. A breach of the skin's protective barrier may thus not be initially noticed. Altered sensory perception may also cause diabetic patients to tolerate the symptoms of early infection for longer before seeking help. ${ }^{9}$ Impaired peripheral vascularity further compounds the effects of impaired immunity. The abovementioned factors place diabetics at risk for developing infections and predisposes diabetic patients to a six times greater risk of experiencing complications. ${ }^{11}$ Our finding that poorly controlled diabetes mellitus is a significant independent risk factor for the development of early complications (OR 4.4; $95 \% \mathrm{Cl}$ $1.12-17.38)$ is in agreement with current literature and remained significant after multivariate analysis. ${ }^{8}$

The frequency of complications seen in patients presenting to private GPs is noteworthy, but with the small number of cases involved it is difficult to draw any conclusions in terms of this finding. A number of the cases that developed complications involved an informal incision and drainage in the consulting rooms, which raises questions around the adequacy of the surgical debridement in this setting. Although simple lancing may decompress superficial collections and relieve pain to some extent, incomplete drainage and recollection is common. Sub-optimal debridement may lead to persistence of an infective nidus and continued soft tissue compromise. Underestimation of the extent of infection, especially in tenosynovitis and deep space infections, may also contribute to the development of complications. Furthermore, the oral antibiotics which would typically be prescribed on an outpatient basis may not have the necessary bioavailability in comparison to parenteral treatment. The finding of increased complications after initial outpatient management emphasises the potential value of optimal surgical conditions, in-patient care, parenteral antibiotics and a surgeon experienced in surgical management of complex hand infections.

Infections resulting from human bites were associated with the development of early complications in our study population and resulted in amputation in three cases. Although the literature suggests that Eikenella corrodens is the most common organism isolated in human bites, ${ }^{3}$ we did not encounter this organism. Polymicrobial infections were seen in $29 \%$ of the human bites in this series, which was comparable to previous studies. ${ }^{11} \mathrm{~A}$ high index of suspicion should be maintained for the presence of associated tendon injury and intra-articular penetration when human bites are encountered.,4 Prompt administration of broad spectrum, parenteral antibiotics and surgical debridement followed by splinting and a low threshold for re-debridement at 48 hours is advised to limit complications. ${ }^{3}$

The bacteriology and antibiotic sensitivity profiles of the causative organisms may vary between institutions and regions; however, the findings in our study are in keeping with those that have previously been reported in the literature. Historically, S. aureus was reported to be responsible for up to $86 \%$ of hand infections. ${ }^{3,16}$ More recent international and local literature suggest that polymicrobial infections are becoming more frequent., 2,3,11 This trend is also reflected in our data with more than a quarter of infections involving Gram-negative or polymicrobial infections. Existing data suggests that diabetics and HIV-positive patients are more commonly affected by polymicrobial, Gram-negative and anaerobic infections. ${ }^{2,5}$ Klebsiella $s p$. was the most frequently isolated Gram-negative organism in our study, and most (67\%) of these cultures were obtained from diabetic patients who were particularly poorly controlled (HbA1c 14.4-15.6). It is important to recognise that there was no growth in $31 \%$ of our cases and factors such as pre-operative antibiotic administration and the quality of specimens taken may have contributed to this. Taking tissue samples for culture, for example, could potentially have increased the yield compared to pus swabs.

The existing literature suggests a multitude of factors that appear to have an influence on complications in hand infections when they are viewed in isolation (on univariate level). To our knowledge this is the first study including HIV-positive and diabetic patients with hand infections that also incorporated a multivariate analysis. This showed that many of the risk factors which are considered to be significant on a univariate level may not remain significant in a multivariate model. Some frequently cited publications may have been underpowered for the reported outcomes and may also fail to account for the complex interplay between multiple risk factors. There are, however, several limitations to this study, principally among which is the relatively small sample size. There was a very small difference in the complication rate between the HIV-positive patients and the control group and thus the study was insufficiently powered to assess HIV as a risk factor for the development of early complications. While the study was sufficiently powered to assess human bites, a larger number of cases would also be required to confirm diabetes as a risk factor. The second important shortcoming is the focus on early complications, the lack of long-term followup and functional outcome data. While functional outcomes were available for a small number of cases, the data was not sufficient to allow analysis. Patients were often followed up at their nearest hospital due to the long distances between referral centres and transport restraints. This may have contributed to inconsistencies in patient attendance and recording of functional outcomes. Future studies involving the prospective enrolment and long-term followup of patients, including the assessment of functional outcomes, are needed to determine all other risk factors for complications following hand infections. 


\section{Conclusion}

Hand infections resulting from human bites and those occurring in diabetic patients with poor glucose control appear to be more prone to the development of early complications. HIV infection was not found to be a significant risk factor in this series. While the majority of infections were still caused by $S$. aureus, a number of Gram-negative and polymicrobial infections were identified. The addition of empiric Gram-negative antibiotic cover should be considered, particularly in high risk patients.

\section{Acknowledgement}

The authors would like to thank Prof Reitze Rodseth for his contributions.

\section{Ethics statement}

Ethical approval was obtained from the University of KwaZulu-Natal BREC ethics committee (BEO14/17), the KwaZulu-Natal Department of Health (KZ_2017RP27_823) and the Ngwelezana Hospital CEO prior to the commencement of data collection.

\section{Declaration}

The authors declare authorship of this article and that they have followed sound scientific research practice. This research is original and does not transgress plagiarism policies

\section{Author contributions}

HV proposed the study concept, study design, data collection, data analysis and manuscript preparation.

LCM contributed to study design, data analysis and manuscript preparation.

PVR contributed to study design, manuscript preparation and co-supervised the study. PDR contributed to conceptualisation, study design, manuscript preparation and supervised the study.

\section{ORCID}

Verhoef H (D) https://orcid.org/0000-0002-6164-4892

Marais LC (DD https://orcid.org/0000-0002-1120-8419

Ryan PV (D) https://orcid.org/0000-0002-0957-6482

Rollinson PD (D) https://orcid.org/0000-0002-2384-7250

\section{References}

1. Koshy JC, Bell B. Hand infections. J Hand Surg Am. 2019 Jan;44(1):46-54.

2. Greyling JF, Visser E, Elliot E. Bacteriology and epidemiology of hand infections. SA Orthop J. 2012;11(1):57-62.

3. McDonald LS, Bavaro MF, Hofmeister EP, Kroonen LT. Hand infections. J Hand Surg Am. 2011;36(8):1403-12.

4. Osterman M, Draeger R, Stern P. Acute hand infections. $J$ Hand Surg Am. 2014;39(8):1628-35.

5. Gonzalez MH, Nikoleit J, Weinzeig N, Pulvirenti J. Upper extremity infections in patients with the human immunodeficiency virus. $J$ Hand Surg Am. 1998;23(2):348-52.

6. McAuliffe JA, Seltzer DG, Hornicek FJ. Upper-extremity infections in patients seropositive for human immunodeficiency virus. $J$ Hand Surg Am. 1997;22(6):1084-90.

7. Glickel SZ. Hand infections in patients with acquired immunodeficiency syndrome. J Hand Surg Am. 1988;13(5):770-75.

8. Schmidt G, Piponov H, Chuang D, Gonzalez M. Hand infections in the immunocompromised patient: an update. $J$ Hand Surg Am. 2019;44(2):144-49.

9. Abbas ZG, Gill GV, Archibald LK. The epidemiology of diabetic limb sepsis: an African perspective. Diabet Med 2002;19(11):895-99.

10. Ching V, Ritz M, Song C, et al. Human immunodeficiency virus infection in an emergency hand service. J Hand Surg Am. 1996;21(4):696-99.

11. Houshian S, Seyedipour S, Wedderkopp N. Epidemiology of bacterial hand infections. Int J Infect Dis. 2006;10(4):315-19.

12. Statistics South Africa [Internet]. Statistical release P0302 mid-year population estimates. Pretoria: Statistics South Africa [updated 25 Aug 2016; cited 16 Oct 2016]. Available from: http:// www.statssa.gov.za/publications/P0302/P03022016.pdf.
13. South African National AIDS Council, National Department of Health [Internet]. Global AIDS response progress report 2012. [Updated 29 Feb 2012; cited 16 Oct 2016]. Available from: http:// files.unaids.org/en/dataanalysis/knowyourresponse/countryprogres sreports/2012countries/ce_ZA_Narrative_Report.pdf.

14. Wynn SW, Elhassan BT, Gonzalez MH. Infections of the hand in the immunocompromised host. J Hand Surg Am. 2004;4(2):121-27.

15. Mandel MA. Immune competence and diabetes mellitus: pyogenic human hand infections. J Hand Surg Am. 1978;3(5):458-61.

16. Stone $\mathrm{NH}$, Hursch $\mathrm{H}$, Humphrey $\mathrm{CR}$, Boswick JA Jr. Empirical selection of antibiotics for hand infections. J Bone Joint Surg Am. 1969;51(5):899-903 